\title{
Association of miR-1251-5p/miR-6892-5p Expression With Clinicopathological Factors in Premenopausal Endometrial Cancer
}

\section{Jing Zhang}

Shaanxi Provincial People's Hospital, Third Affiliated Hospital of Xi 'an Jiaotong University

\section{Bin Li}

Shaanxi Provincial People's Hospital, Third Affiliated Hospital of Xi 'an Jiaotong University

\section{Yang Liu}

Shaanxi Provincial People's Hospital, Third Affiliated Hospital of Xi 'an Jiaotong University

\section{Fei Wang}

Shaanxi Provincial People's Hospital, Third Affiliated Hospital of Xi 'an Jiaotong University

\section{Yu Zhang}

Shaanxi Provincial People's Hospital, Third Affiliated Hospital of Xi 'an Jiaotong University

\section{Lu Gan ( $\nabla$ ganlu-789@163.com )}

Shaanxi Provincial People's Hospital, Third Affiliated Hospital of Xi 'an Jiaotong University

\section{Research Article}

Keywords: premenopausal endometrial cancer, differentially expressed microRNAs, next-generation sequencing, miR-1251-5p, miR-6892-5p

Posted Date: September 8th, 2021

DOl: https://doi.org/10.21203/rs.3.rs-877847/v1

License: (c) (1) This work is licensed under a Creative Commons Attribution 4.0 International License. Read Full License 


\section{Abstract \\ Background}

This study is aim to profile the differentially expressed microRNAs (DEMs) of premenopausal endometrial cancer (EC), identify their target genes and understand their roles in carcinogenesis.

\section{Methods}

Next-generation sequencing (NGS) was performed on 3 premenopausal EC and 3 premenopausal normal endometrial tissues. Selection of candidate miRNAs and subsequent validation were performed by qRTPCR on 20 premenopausal EC, 30 premenopausal normal endometrial and 40 postmenopausal EC samples. The relationship between DEMs and clinical characteristics was analyzed. Moreover, bioinformatic software programs and databases were applied to predict miRNA target genes, molecular functions, and signaling pathways.

\section{Results}

136 upregulated and 131 downregulated DEMs were identified. The expression of miR-1251-5p was highly upregulated in premenopausal EC samples compared with premenopausal normal endometrial samples and significantly downregulated compared with postmenopausal EC samples. The expression of miR-6892-5p was highly upregulated in premenopausal EC samples compared with premenopausal normal endometrial samples and postmenopausal EC samples. In the premenopausal EC group, miR1251-5p expression was closely correlated with menarche age, number of pregnancies, tumor grading, myometrial infiltration and lymph node metastasis; miR-6892-5p expression was closely correlated with $\mathrm{BMI}$, hypertension, tumor grading, and metastasis.

\section{Conclusions}

miR-1251-5p and miR-6892-5p may play important roles in tumorigenesis progression of premenopausal EC.

\section{Introduction}

Endometrial cancer (EC) is one of the most common cancers of the female reproductive system. The incidence has risen by an approximate 50\% since the early 1990s, and this trend is set to continue with a predicted incidence of $33 / 100,000$ by $2035^{[1]}$. EC occurs predominantly among postmenopausal women, with a mean diagnosed age of 61 years; however, premenopausal EC is not rare. Approximately $20 \%$ of EC patients are premenopausal women, $5-30 \%$ are diagnosed before the age of 50 years old and $5 \%$ are 
diagnosed before the age of 40 years old ${ }^{[2-3]}$. Interestingly, some recent studies have reported that Asian women, including Asian native women and Asian immigrant women, are diagnosed with EC at a younger age than non-Asian women ${ }^{[4]}$, and the proportion of Chinese women diagnosed with EC before menopause $(38 \%)$ is higher than that of Western countries $(<25 \%)^{[5]}$. Although the incidence of EC in young women has dramatically increased due to early-onset obesity and hyperinsulinemia in the last decade, there have been few studies on the clinical characteristics and pathogenesis related to premenopausal EC.

MicroRNAs (miRNAs) are small, single-stranded, noncoding RNAs composed of $19 \sim 25$ nucleotides that play major roles in cell proliferation, cell death, metabolism, stem cell maintenance and differentiation and disease development in post-transcriptional gene regulation ${ }^{[7]}$. Understanding how miRNAs act as master regulators in these important networks implicated in EC initiation and progression will provide new insight into therapeutic and diagnostic approaches.

Therefore, in this study, we aimed to identify differentially expressed miRNAs (DEMs) in premenopausal EC by next-generation sequencing (NGS) and qRT-PCR techniques. By identifying miRNA-gene expression signatures in premenopausal EC, we may provide new perspectives for the development of novel diagnostic methods, prognostic prediction tools, and therapeutic strategies for EC in younger women.

\section{Results}

\section{Analysis of DEMs}

A total of 2551 miRNAs were obtained, of which 1711 were known miRNAs and 840 were newly predicted miRNAs. In total, 267 miRNAs with a threshold of $\mathrm{P}<0.05$ and $|\log 2 \mathrm{FC}|$ were considered significantly $\mathrm{DE}$, 136 of which were upregulated and 131 of which were downregulated. The top 20 upregulated genes and top 20 downregulated genes are shown in Table S1. The differences in miRNA expression levels between the two groups of samples and the statistical significance of the differences were observed via the MA map cluster diagram and volcano plot (Figure 1).

\section{Known DEMs in EC Identified by Sequencing and qRT-PCR}

The qRT-PCR results showed that although the melt curves of miR-6744-3p, hsa-miR-1258 and hsa-miR$371 a-5 p$ displayed no specificity, those of the other 7 miRNAs were all specific. Through additional analysis, we found that the above 7 miRNAs had significant differences in expression between normal endometrial tissues and EC (including premenopausal EC and postmenopausal EC) tissues; except for miR-133a-5p, the expression of the other 6 miRNAs between premenopausal EC and normal endometrial tissues was also significantly different, while there were three significant DEMs (miR-1251-5p, miR-133a$5 p$ and miR-6892-5p) between the premenopausal EC and postmenopausal EC groups, and the expression of two genes (miR-1251-5p and miR-6892-5p) was significantly different between premenopausal EC tissues and normal endometrium and between premenopausal EC and postmenopausal EC tissues (Table S2, Figure 2). 


\section{Target Gene Prediction}

To explore the biological function of the DEMs identified in this analysis, computational analysis was performed using two independent experimentally confirmed databases, miRanda and TargetScan, to analyze the target RNAs of miR-1251-5p and miR-6892-5p (Table S3).

\section{Functional and Pathway Enrichment Analysis of DEMs}

To further gain insights into the biological roles of DEMs in premenopausal EC, we performed GO term and KEGG enrichment analyses for 267 DEMs. The GO term enrichment results demonstrated that in the biological process analysis, these genes were mainly enriched for cellular process, single-organism process and biological regulation; in the cellular component analysis, these genes were enriched for cell, cell part, and organelle; and in the molecular function analysis, these genes were mainly enriched for binding and catalytic activity (Figure S1).The top 10 significantly enriched GO categories of biological processes, cellular components, and molecular functions are listed in Figures 3-1, 2, and 3, and KEGG pathway analysis of the top 10 significantly enriched signaling pathways revealed that the most significantly enriched pathway was pathways in cancer (Figures 3-4).

\section{DEMs and Clinical Factors}

In the premenopausal EC group, the expression of miR-1251-5P was significantly different in menarche age $(P=0.017)$, number of pregnancies $(P=0.000)$, tumor cell differentiation level $(P=0.031)$, myometrial infiltration $(P=0.046)$, and lymph node metastasis $(P=0.011)$, and the expression of miR-6892-5P was significantly different in $B M I(P=0.004)$, hypertension $(P=0.006)$, tumor cell differentiation level $(P=0.006)$, and metastasis (including cervical uteri, lymph node and other organ metastases) $(P=0.005)$. In the postmenopausal EC group, there was no significant difference in miR-1251-5p expression in any of the factors above except diabetes $(P=0.031)$, and there was no significant difference in miR-6892-5p expression in any of the factors above except FIGO stage $(P=0.040)($ Tables 1,2$)$.

Comparisons of the clinical and pathological characteristics between premenopausal and postmenopausal EC patients showed that the age of menarche of premenopausal EC patients was significantly earlier than that of postmenopausal EC patients, and the proportion of patients with irregular menstrual cycle and obesity was significantly higher than that of postmenopausal EC patients. However, the proportion of postmenopausal EC patients with hypertension and poor tissue differentiation was significantly higher than that of premenopausal EC patients (Table 3).

Sixty EC patients were followed up to 2020-10, with the longest follow-up time of 34 months and the shortest follow-up time of 12 months. There were 3 cases of recurrent disease, all of which were postmenopausal EC. One patient (stage IB, G3) who did not undergo postoperative radiotherapy due to postoperative urine retention refused treatment after lung metastasis occurred 13 months after surgery (death). One patient (stage IB, G1) had rectal metastasis recurrence 17 months after surgery and one patient (stage IIIC1, G1) developed distant metastasis and recurrence to the peritoneum and omentum 14 
months after surgery. Both of these patients are currently under treatment. There was no correlation between recurrence and miR-1251-5p or miR-6892-5p expression levels $(P=0.501, P=956)$.

\section{Discussion}

It is well known that $80 \%-85 \%$ of ECs are endometrioid adenocarcinomas associated with estrogen dependence. Non-resistant estrogen exposure is an important risk factor; long-term exposure leads to persistent endometrial hyperplasia and even progression to cancer ${ }^{[8]}$. Before menopause, estrogen and progesterone are primarily synthesized in the ovaries, although estrogens are also produced via the peripheral testosterone aromatization, especially in obese women. During the transition to the perimenopausal period, ovarian failure gradually leads to a shift in the balance of these two hormones toward more unopposed extraglandular production of estrogen in the body, which may lead to differences in risk factors and pathogenesis between premenopausal and postmenopausal women. In recent years, accumulating evidence has shown that menopause and older age might be a risk factor for malignancy of endometrial polyps and abnormal uterine bleeding $(\mathrm{AUB})^{[9-10]}$, whereas premenopausal EC is more closely associated with obesity (Michelle R. Wise et al. 2016) ${ }^{[11]}$, polycystic ovary syndrome (PCOS), nulliparity (Pamela T. Soliman et al. 2005) ${ }^{[12]}$, and diabetes (Mu Nan 2018,Luca Giannella 2019) $)^{[13-14]}$. Our study confirmed the findings that among premenopausal EC patients, the proportion of obese and irregular menstrual cycle patients was significantly higher than that of postmenopausal EC patients. These studies suggest that in addition to hormonal factors, insulin also plays a significant role in the carcinogenesis of EC in premenopausal women. In addition, the differences in pathologic characteristics and prognosis for premenopausal EC and postmenopausal EC vary in different studies. Several previous studies have reported an incidence of concurrent ovarian cancer in young EC women ranging from 7$29 \%$ and recommended caution when considering ovarian preservation in young EC patients ${ }^{[12,15-16]}$. However, other studies have shown that young women with EC often have low-grade, early-stage tumors and have a more favorable prognosis than older patients and suggest that ovarian preservation in premenopausal women with early-stage EC may be safe and not associated with an increase in cancerrelated mortality ${ }^{[2,17-18]}$. Our study found that premenopausal $E C$ had good grading $(P=0.004)$ and a lower recurrence rate ( 0 vs. $5 \%$ ) than postmenopausal EC, although there was no statistically significant difference in the recurrence rate between the two groups due to the small number of cases and short follow-up time. This discrepancy may be due to differences in sample sizes, the characteristics of patients, and study regions. These studies identified differences in ECs between premenopausal and postmenopausal women, suggesting that there may be differences in tumor biology between the two groups.

Abnormal miRNA expression leads to the occurrence of certain diseases and even carcinogenesis ${ }^{[19-22]}$, including endometrial cancer ${ }^{[23-25]}$. Some studies have found that miRNA expression is associated with prognostic factors, including lymph node involvement, lymphovascular space invasion (LVSI), recurrencefree survival (RFS) and overall survival (OS) ${ }^{[26-27]}$. miRNAs are particularly attractive candidates as biomarkers for cancer diagnosis and treatment because of their potential role in controlling cell growth 
and differentiation ${ }^{[19-22,28-29]}$. This study investigated differences in the miRNA gene profile for premenopausal EC by next-generation sequencing techniques and identified a total of $267 \mathrm{DEMs}$ between premenopausal EC and normal endometrial tissues. The majority of the DEMs had unknown functions. miRNA-1251-5p expression was highly upregulated in premenopausal EC samples compared with premenopausal normal endometrial samples and significantly downregulated compared with that in postmenopausal EC samples. miR-6892-5p expression was highly upregulated in premenopausal EC samples compared with premenopausal normal endometrial and postmenopausal EC samples. To our knowledge, this is the first report on the miRNA expression profile of premenopausal EC. Improved knowledge of the miRNA expression profile may explain molecular mechanisms involved in premenopausal EC and may serve as a basis for the development of diagnostic and prognostic indicators or the identification of potential new therapeutic targets.

miR-1251-5p is located on chromosome 12q23.1. Previously, some studies showed that miR-1251-5p expression was downregulated in stomach carcinoma cells and clear cell renal cell carcinoma ${ }^{[30]}$. Other studies found that miR-1251-5p, a tumor driver, promotes malignant behavior and autophagy of ovarian cancer cells by targeting the tumor suppressor gene TBCC and promotes tumor growth and metastasis of liver cancer by targeting AKAP12 ${ }^{[32]}$. These studies indicate that miR-1251-5p biological functions in different carcinomas are diverse. Our experiments also show that miRNA-1251-5p expression was highly upregulated compared with that in premenopausal normal endometrial samples and significantly downregulated compared with that in postmenopausal EC samples. Additionally, in the pre-ca group, its expression was significantly upregulated in cancer tissues of patients with early menarche, low fertility, poor differentiation and deep muscular invasion compared with patients with late menarche, well differentiated tumors and shallow muscular invasion, but no such associations (except diabetes) were observed in postmenopausal EC, suggesting that miR-1251-5p may be a key gene affecting the occurrence and invasion of premenopausal EC. However, miR-1251-5p expression was significantly decreased in premenopausal EC patients with lymph node positivity compared with negative patients, which may be related to the relatively small number of lymph node metastases. These relationships require further verification with a large sample.

There have been no reports on miR-6892-5p. This study showed that miR-6892-5p expression was highly upregulated in premenopausal EC samples compared premenopausal normal endometrial and postmenopausal EC samples. In the premenopausal EC group, miR-6892-5p expression was closely correlated with tumor cell differentiation level and metastasis. We used miRanda and TargetScan to analyze the target RNAs of miR-6892-5p. Some of these target genes, such as HEATR1 and FRK, have been reported to act as tumor suppressor genes in different tumors. Some of these target genes, including CCDC80, NDUFB3 and NDUFB5, play an important protective role in glycolipid metabolism ${ }^{[38-}$ 40]. Our study found that miR-6892-5p expression was significantly different in the endometrial cancer tissues of premenopausal EC between obese, overweight and normal populations. Based on these findings, it can be concluded that miR-6892-5p may be associated with the tissue characteristics and carcinogenesis of premenopausal EC by regulating insulin metabolism and affecting fat distribution 
through these target genes. The target RNAs and their related functions need to be further studied in subsequent experiments.

\section{Conclusions}

In conclusion, miR-1251-5p and miR-6892-5p may play important roles in the occurrence and development of premenopausal EC. However, due to the relatively limited number of sequencing samples and verification samples in this experiment, these findings need to be further verified in larger samples. Their target RNAs and signaling pathways related to premenopausal EC also need to be further studied.

\section{Materials And Methods}

\section{Patient Samples}

The study was approved by the Ethics Committee of Shaanxi Provincial People's Hospital and was performed according to the principles of the Declaration of Helsinki. All experiments were performed in accordance with relevant guidelines and regulations, and informed consent was obtained from the participants. Tumor tissue samples were obtained from complete staging of endometrial carcinoma (bilateral salpingo-oophorectomy and pelvic and para-aortic lymphadenectomy) with previously untreated EC and were divided into premenopausal (pre-ca group) and postmenopausal (post-ca group) groups according to whether the patients were menopausal or not. All samples were histologically classified and graded by a clinical pathologist according to WHO guidelines. Healthy endometrial tissues ( $\mathrm{N}$ group) were collected as negative controls from patients undergoing hysterectomy or curettage procedures for benign problems, such as endometrial hyperplasia and uterine myoma, and did not have a diagnosis of any type of cancer or prior cancer history.

Patient set 1 used for next-generation sequencing consisted of samples from 6 individuals (Table S4): 3 premenopausal EC tissue samples and 3 premenopausal normal endometrial tissue samples. For validation of the sequencing results, RNA samples from a second series of tumor and healthy tissues were prepared to examine miRNA expression via quantitative real-time PCR (qRT-PCR). Patient set 2 consisted of 90 samples (20 cases in the pre-ca group, 40 cases in the post-ca group and 30 cases in the N group) (Table S5).

All samples were collected at the Department of Gynecology of Shaanxi Provincial People's Hospital, First Affiliated Hospital of Xi 'an Jiaotong University and Department of Female Tumor of Shaanxi Provincial Cancer Hospital from January 2017 to September 2019. Tissues were immediately snap-frozen in liquid nitrogen and then transferred to a $-80^{\circ} \mathrm{C}$ freezer following surgery.

\section{RNA Extraction, Small RNA Library Construction and Next-Generation Sequencing}

Total RNA was extracted using a NAfast1000 Total RNA extraction kit (Piomeer, Xian, China) according to the manufacturer's instructions. RNA concentration and purity was quantified using a NanoDrop 2000 
Spectrophotometer (Thermo Fisher Scientific, Wilmington, DE) at O.D. $260 \mathrm{~nm}$. RNA integrity was assessed using the RNA Nano 6000 Assay Kit of the Agilent and Bioanalyzer 2100 System (Agilent Technologies, CA, USA) and RNA integrity number (RIN) $>8.0$ is the cutoff for RNA sequencing.

Sequencing libraries were generated according to the manufacturer's instructions using the NEBNext UltraTM small RNA Sample Library Prep Kit for Illumina (NEB, USA), and the index codes were added to the property sequence of each sample. Truseq PE Cluster KITV3-CBOT-HS (Illumina) was used to cluster the index-coded samples on the СВОT Cluster Generation System according to the manufacturer's instructions. After the cluster was generated, the library was prepared for sequencing, and reads were generated on the Illumina platform. Sequencing was performed at Biomarker Technologies (Beijing).

\section{Bioinformatics and Data Processing}

DEMs between premenopausal EC specimens and premenopausal normal endometrial tissue specimens were identified via the edgeR package ${ }^{[3]}$. The resulting false discovery rate (FDR) was adjusted using the posterior probability of being DE (PPDE). An adjusted $P$-value $<0.05$ and a $|F C| \geq 1.5$ were the thresholds for significant differential expression.

\section{Functional Enrichment Analysis of DEM Target Genes}

The GO database is a structured standard biological annotation system containing three major branches, namely, the biological process, molecular function and cellular component branches. KEGG is a database resource to understand the advanced functions and utilities of biological systems. GO enrichment and KEGG pathway analyses of the target genes of DEMs were implemented by the clusterProfiler $\mathrm{R}$ package.

\section{Detection of DEMs}

The quantification of miRNAs was performed by a two-step reaction using an Evo M-MLV RT Kit for qPCR (AG, China, Hunan) and a SYBR Green Premix Pro Taq HS qPCR kit (Pioneer, China, Xi'an) for validating the expression of miRNAs(Table S6). qPCR was carried out using an ABI7500 fluorescence quantitative PCR instrument ( $A B I, U S A)$. Cycling conditions were as follows: $95^{\circ} \mathrm{C}$ for $5 \mathrm{~min}$, followed by 40 cycles at $\left[95^{\circ} \mathrm{C}\right.$ for $20 \mathrm{sec}, 50^{\circ} \mathrm{C}$ for $30 \mathrm{sec}, 72^{\circ} \mathrm{C}$ for $30 \mathrm{sec}$, and $95^{\circ} \mathrm{C}$ for $15 \mathrm{sec}$, $50^{\circ} \mathrm{C}$ for $1 \mathrm{~min}$, and $95^{\circ} \mathrm{C}$ for $30 \mathrm{~s}$. All reactions were performed in triplicate. All samples were analyzed using the endogenous reference RNA RNU6B. The fold changes in miRNA expression were calculated using the $2^{-\Delta \Delta \mathrm{Ct}}$ method, where $\Delta \Delta \mathrm{Ct}=$

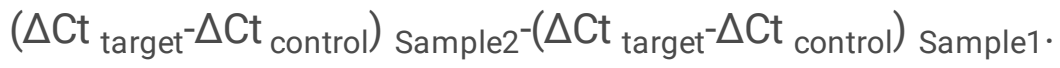

\section{Statistical Analyses}

The miRNA expression data were statistically analyzed using the Statistical Product and Service Solutions (SPSS) Statistical Package, Version 23.0 (Chicago, Illinois). All measurement data are expressed as the mean+s.d., and $\chi^{2}$ test was used for counting data. Student's t-test or the Mann-Whitney $U$ test was used to compare two groups, according to the result of the Shapiro-Wilk normality test. All statistical tests were two-sided, and P-values $<0.05$ were considered significant. 


\section{Declarations}

\section{Acknowledgments}

The authors would like to thank Biomarker Technologies (Beijing) for excellent technical assistance.

\section{Funding}

This study was funded by Grants of the National Natural Science Foundation of China (Funding organization: the National Natural Science, P.R. China, grant number 81801414), the Natural Science Youth Fund Foundation of Shaanxi Province (Funding organization: the Health Department of Shaanxi Province, P.R. China, grant number 2021JQ-912), the Natural Science Youth Fund Foundation of Shaanxi Province (Funding organization: the Health Department of Shaanxi Province, P.R. China, grant number 2021JQ-913), and the Science and Technology Talent Support Program of Shaanxi Provincial People's Hospital in 2021 (Funding organization: Shaanxi Provincial People's Hospital, P.R. China, grant number 2021JY-34).

\section{Affiliations}

Shaanxi Provincial People's Hospital, Third Affiliated Hospital of Xi 'an Jiaotong University, Shaanxi Xian 710068, China

Jing Zhang, Bin Li, Yang Liu, Fei Wang, Yu Zhang, Lu Gan

\section{Contributions}

$\mathrm{JZ}$, principal author, manuscript writing and statistical analyses analysis; $\mathrm{BL}$, experiment operation; $\mathrm{YL}$, directed the operation of the experiment; FW, perform the analysis and participated in constructive discussions; $Y Z$, specimens collection and perform the data analyses; LG, corresponding author, contributed to the conception of the study, manuscript writing supervision and statistical analyses supervision. All authors have read and agreed to the published version of the manuscript.

\section{Corresponding authors}

Correspondence to Lu Gan.

\section{Conflict of Interest statement}

The authors declare that there are no conflicts of interest.

\section{Availability of data and materials}

The datasets used and analyzed during the current study are available from the corresponding author upon reasonable request. 
Declarations Ethics approval and consent to participate

This study was approved by the Ethics Committee of Shaanxi Provincial People's Hospital and was conducted in accordance with the principles expressed in the Helsinki Declaration.

\section{Consent for publication}

Not applicable.

\section{References}

1. Hutt Suzanna,Tailor Anil,Ellis Patricia, et al. The role of biomarkers in endometrial cancer and hyperplasia: a literature review.[J].Acta Oncol, 2019, 58: 342-352. (http://doi:10.1080/0284186X.2018.1540886)

2. Zhang, X. Li Fengshuang,Zhu Prof Linzhong.Fyn-related kinase expression predicts favorable prognosis in patients with cervical cancer and suppresses malignant progression by regulating migration and invasion.[. J].Biomed Pharmacother, 84, 270-276 https://doi.org/http://doi:10.1016/j.biopha.2016.09.042 (2016).

3. Goel Raghuveera Kumar,Lukong Kiven Erique,Understanding the cellular roles of Fyn-related kinase (FRK): implications in cancer biology.[J].Cancer Metastasis Rev, 2016, 35:179-99. (http://doi:10.1007/s10555-016-9623-3)

4. Zhen, H. L. W. G. et al. Activation of STAT1 by the FRK tyrosine kinase is associated with human glioma growth.[J].J Neurooncol, 2019, 143: 35-47. (http://doi:10.1007/s11060-019-03143-w)

5. Grill Jessica, I. et al. Neumann Jens,Herbst Andreas, Loss of DR01/CCDC80 results in obesity and promotes adipocyte differentiation.[J].Mol Cell Endocrinol, 2017, 439: 286-296. (http://doi:10.1016/j.mce.2016.09.014)

6. Kang Ki Woon,Kim Ok Soon,Chin Jung Yeon. et al. Diastolic Dysfunction Induced by a High-Fat Diet Is Associated with Mitochondrial Abnormality and Adenosine Triphosphate Levels in Rats.[J]. Endocrinol Metab (Seoul), 30, 557-568 https://doi.org/10.3803/EnM.2015.30.4.557 (2015). http://doi

7. Sparks Lauren, M. et al. A high-fat diet coordinately downregulates genes required for mitochondrial oxidative phosphorylation in skeletal muscle.[J].Diabetes, 2005, 54:1926-33. (http://doi:10.2337/diabetes.54.7.1926)

8. Key T J,Pike M C,The dose-effect relationship between 'unopposed' oestrogens and endometrial mitotic rate: its central role in explaining and predicting endometrial cancer risk. [J] . Br J Cancer, 1988, 57: 205 - 12. (http://doi:10.1038/bjc.1988.44)

9. Costa-Paiva Lucia,Godoy Carlos E,Antunes Armando et al. Risk of malignancy in endometrial polyps in premenopausal and postmenopausal women according to clinicopathologic characteristics.[J] .Menopause, 2011, 18: 1278-82. (http://doi:10.1097/gme.0b013e31821e23a1) 
10. Pennant M E,Mehta R,Moody P, et al. Premenopausal abnormal uterine bleeding and risk of endometrial cancer.[J] .BJOG, 2017, 124: 404-411. (http://doi:10.1111/1471-0528.14385)

11. Wise Michelle R,Jordan Vanessa,Lagas Alice, et al. Obesity and endometrial hyperplasia and cancer in premenopausal women: A systematic review.[J] .Am J Obstet Gynecol, 2016, 214: 689.e1-689.e17. (http://doi:10.1016/j.ajog.2016.01.175)

12. Soliman Pamela T,Oh Jonathan C,Schmeler Kathleen M, et al. Risk factors for young premenopausal women with endometrial cancer.[J] .Obstet Gynecol, 2005, 105: 575 - 80.

(http://doi:10.1097/01.AOG.0000154151.14516.f7)

13. Mu Nan,Dong Mei,Liu Chunyan, et al. Association between preoperative serum insulin levels and lymph node metastasis in endometrial cancer-a prospective cohort study.[J] .Cancer Med, 2018, 7: 1519-1527. (http://doi:10.1002/cam4.1391)

14. Giannella Luca,Cerami Lillo Bruno,Setti Tiziano et al. Prediction of Endometrial Hyperplasia and Cancer among Premenopausal Women with Abnormal Uterine Bleeding.[J] .Biomed Res Int, 2019, 2019: 8598152. (http://doi:10.1155/2019/8598152)

15. Evans-Metcalf E R,Brooks S E,Reale F R, et al. Profile of women 45 years of age and younger with endometrial cancer.[J] .Obstet Gynecol, 1998, 91: 349 - 54. (http://doi:10.1016/s00297844(97)00668-6)

16. Wright Jason D,Jorge Soledad,Tergas Ana I, et al. Utilization and Outcomes of Ovarian Conservation in Premenopausal Women With Endometrial Cancer.[J] .Obstet Gynecol, 2016, 127: 101-108. (http://doi:10.1097/AOG.0000000000001181)

17. Gressel Gregory M,Parkash Vinita,Pal Lubna,Management options and fertility-preserving therapy for premenopausal endometrial hyperplasia and early-stage endometrial cancer.[J] .Int J Gynaecol Obstet, 2015, 131: 234-9.(http://doi:10.1016/j.ijgo.2015.06.031)

18. Gonthier Clémentine,Douhnai Daria,Koskas Martin,Lymph node metastasis probability in young patients eligible for conservative management of endometrial cancer.[J] .Gynecol Oncol, 2020, 157: 131-135. (http://doi:10.1016/j.ygyno.2020.02.021)

19. Chen Xi,Ba Yi,Ma Lijia, et al. Characterization of microRNAs in serum: a novel class of biomarkers for diagnosis of cancer and other diseases.[J] .Cell Res, 2008, 18: 997-1006. (http://doi:10.1038/cr.2008.282)

20. Cortez Maria Angelica,Anfossi Simone,Ramapriyan Rishab et al. Role of miRNAs in immune responses and immunotherapy in cancer.[J] .Genes Chromosomes Cancer, 2019, 58: 244-253. (http://doi:10.1002/gcc.22725)

21. Pardini Barbara,Sabo Alexandru Anton,Birolo Giovanni et al. Noncoding RNAs in Extracellular Fluids as Cancer Biomarkers: The New Frontier of Liquid Biopsies.[J] .Cancers (Basel), 2019, 11: undefined. (http://doi:10.3390/cancers11081170)

22. Pardini Barbara,Sabo Alexandru Anton,Birolo Giovanni, et al. Noncoding RNAs in Extracellular Fluids as Cancer Biomarkers: The New Frontier of Liquid Biopsies.[J] .Cancers (Basel), 2019, 11: undefined. (http://doi:10.3390/cancers11081170) 
23. Eismann Julia,Hirschfeld Marc,Erbes Thalia, et al. Hypoxia- and acidosis-driven aberrations of secreted microRNAs in endometrial cancer in vitro.[J] .Oncol Rep, 2017, 38: 993-1004. (http://doi:10.3892/or.2017.5717)

24. Ma Jian,Li Da,Kong Fan-Fei, et al. miR-302a-5p/367-3p-HMGA2 axis regulates malignant processes during endometrial cancer development.[J] .J Exp Clin Cancer Res, 2018, 37: 19. (http://doi:10.1186/s13046-018-0686-6)

25. Liu JinHui,Li ChunYu,Jiang Yi, et al. Tumor-suppressor role of miR-139-5p in endometrial cancer.[J] .Cancer Cell Int, 2018, 18: 51. (http://doi:10.1186/s12935-018-0545-8)

26. Wilczynski Milosz,Senderowska Daria,Krawczyk Tomasz, et al. MiRNAs in endometrioid endometrial cancer metastatic loci derived from positive lymph nodes.[J] .Acta Obstet Gynecol Scand, 2020, 99 : 1085-1091. (http://doi:10.1111/aogs.13833)

27. Wang Yue,Xu Mu,Yang Qing,A six-microRNA signature predicts survival of patients with uterine corpus endometrial carcinoma.[J] .Curr Probl Cancer, 2019, 43: 167-176.

(http://doi:10.1016/j.currproblcancer.2018.02.002)

28. Delangle Romain,De Foucher Tiphaine,Larsen Annette K, et al. The Use of microRNAs in the Management of Endometrial Cancer: A Meta-Analysis.[J] .Cancers (Basel), 2019, 11: undefined. (http://doi:10.3390/cancers11060832)

29. Liang Bin,Zhao Jianying,Wang Xuan,A three-microRNA signature as a diagnostic and prognostic marker in clear cell renal cancer: An In Silico analysis.[J] .PLoS ONE, 2017, 12: e0180660.

30. Bibi Fehmida,Naseer Muhammad I,Alvi Sana Akhtar, et al. microRNA analysis of gastric cancer patients from Saudi Arabian population.[J] .BMC Genomics, 2016, 17: 751

31. Shao Yang,Liu Xiaomin,Meng Jiao, et al. MicroRNA-1251-5p Promotes Carcinogenesis and Autophagy via Targeting the Tumor Suppressor TBCC in Ovarian Cancer Cells.[J] .Mol Ther, 2019, 27: 1653-1664. (http://doi:10.1016/j.ymthe.2019.06.005)

32. Han Shaoshan,Wang Liang,Sun Liankang, et al. MicroRNA-1251-5p promotes tumor growth and metastasis of hepatocellular carcinoma by targeting AKAP12.[J] .Biomed Pharmacother, 2020, 122 : 109754. (http://doi:10.1016/j.biopha.2019.109754)

33. Liu Tongzheng,Fang Yuan,Zhang Haoxing, et al. HEATR1 Negatively Regulates Akt to Help Sensitize Pancreatic Cancer Cells to Chemotherapy.[J] .Cancer Res, 2016, 76: 572 - 81. (http://doi:10.1158/0008-5472.CAN-15-0671)

34. Berclaz G,Altermatt $\mathrm{H}$ J,Rohrbach V, et al. Hormone-dependent nuclear localization of the tyrosine kinase iyk in the normal human breast epithelium and loss of expression during carcinogenesis.[J] .Int J Cancer, 2000, 85: 889 - 94. (http://doi:10.1002/(sici)1097-0215(20000315)85:6<889::aidijc25>3.0.co;2-4)

35. Zhang Xiaofeng,Li Fengshuang,Zhu Prof Linzhong.Fyn-related kinase expression predicts favorable prognosis in patients with cervical cancer and suppresses malignant progression by regulating migration and invasion.[J].Biomed Pharmacother, 2016, 84: 270-276. (http://doi:10.1016/j.biopha.2016.09.042) 
36. Goel Raghuveera Kumar,Lukong Kiven Erique,Understanding the cellular roles of Fyn-related kinase (FRK): implications in cancer biology.[J] .Cancer Metastasis Rev, 2016, 35: 179 - 99.

(http://doi:10.1007/s10555-016-9623-3)

37. Hua Lei,Wang Guanghui,Wang Zhen, et al. Activation of STAT1 by the FRK tyrosine kinase is associated with human glioma growth.[J] .J Neurooncol, 2019, 143: 35-47.

(http://doi:10.1007/s11060-019-03143-w)

38. Grill Jessica I,Neumann Jens,Herbst Andreas, et al. Loss of DR01/CCDC80 results in obesity and promotes adipocyte differentiation.[J] .Mol Cell Endocrinol, 2017, 439: 286-296.

(http://doi:10.1016/j.mce.2016.09.014)

39. Kang Ki Woon,Kim Ok Soon,Chin Jung Yeon, et al. Diastolic Dysfunction Induced by a High-Fat Diet Is Associated with Mitochondrial Abnormality and Adenosine Triphosphate Levels in Rats.[J] .Endocrinol Metab (Seoul), 2015, 30: 557 - 68. (http://doi:10.3803/EnM.2015.30.4.557)

40. Sparks Lauren M,Xie Hui,Koza Robert A, et al. A high-fat diet coordinately downregulates genes required for mitochondrial oxidative phosphorylation in skeletal muscle.[J] .Diabetes, 2005, 54: 192633. (http://doi:10.2337/diabetes.54.7.1926)

\section{Tables}


Table 1

Relationship between miR-1251-5p expression and clinic pathological features of EC patients $(\mathrm{X} \pm \mathrm{s})$

Variables miR-1251-5p

Premenopausal EC

Postmenopausal EC

Samples Relative expression $\quad \mathrm{P} \quad$ Samples $\quad$ Relative expression $\quad \mathrm{P}$

Age at menarche ( years)

\begin{tabular}{|c|c|c|c|c|c|}
\hline$\leq 12$ & 8 & $\begin{array}{l}6.76 \times 10^{-3} \pm \\
1.56 \times 10^{-2}\end{array}$ & 0.017 & 9 & $\begin{array}{l}1.63 \times 10^{-2} \pm \\
2.20 \times 10^{-2}\end{array}$ \\
\hline $\begin{array}{l}>12 \sim \leq \\
14\end{array}$ & 8 & $\begin{array}{l}1.75 \times 10^{-2} \pm \\
1.46 \times 10^{-2}\end{array}$ & & 9 & $\begin{array}{l}9.42 \times 10^{-3} \pm \\
6.80 \times 10^{-3}\end{array}$ \\
\hline$>14$ & 4 & $\begin{array}{l}1.37 \times 10^{-3} \pm \\
1.54 \times 10^{-3}\end{array}$ & & 22 & $\begin{array}{l}2.14 \times 10^{-2}{ }_{ \pm} \\
2.48 \times 10^{-2}\end{array}$ \\
\hline
\end{tabular}

Menstrual cycle

$\begin{array}{lllll}\text { Regularity } 8 & 9.25 \times 10^{-3_{ \pm}} & 0.678 & 40 & \\ & & & & \\ & & & & \end{array}$

$\begin{array}{llll}\text { Irregularity } & 12 & 7.25 \times 10^{-3} \pm 1.41 \times 10^{-} & 0\end{array}$

$\mathrm{BMI}$

\begin{tabular}{|c|c|c|c|c|c|}
\hline$<24$ & 3 & $\begin{array}{l}6.62 \times 10^{-3} \pm \\
6.79 \times 10^{-3}\end{array}$ & 0.094 & 10 & $\begin{array}{l}2.49 \times 10^{-2} \pm \\
2.62 \times 10^{-2}\end{array}$ \\
\hline $\begin{array}{l}\geq 24 \sim< \\
28\end{array}$ & 4 & $\begin{array}{l}1.76 \times 10^{-2} \pm \\
1.01 \times 10^{-2}\end{array}$ & & 27 & $\begin{array}{l}1.42 \times 10^{-2} \pm \\
1.85 \times 10^{-2}\end{array}$ \\
\hline$\geq 28$ & 13 & $\begin{array}{l}8.37 \times 10^{-3} \pm \\
1.67 \times 10^{-2}\end{array}$ & & 3 & $\begin{array}{l}2.33 \times 10^{-2}{ }_{ \pm} \\
3.27 \times 10^{-3}\end{array}$ \\
\hline
\end{tabular}

Pregnancies

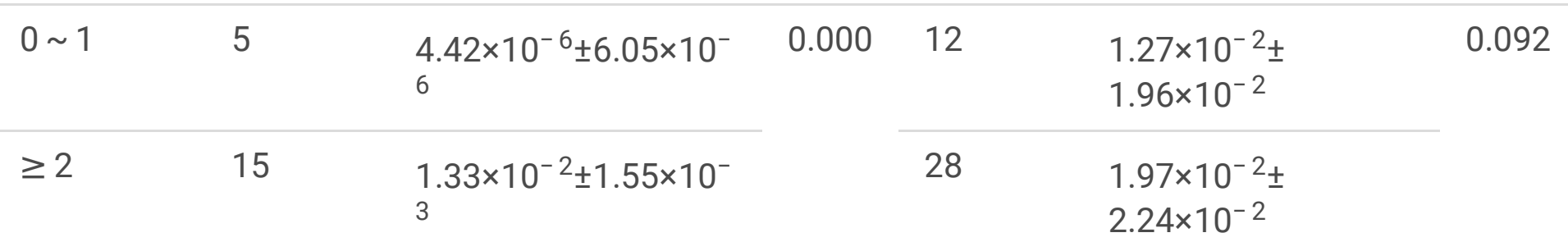

Family history of cancer

(Note: * The menstrual cycle of all the postmenopausal women is regular. Mann-Whitney $\mathrm{U}$ test) 


\begin{tabular}{|c|c|c|c|c|c|c|}
\hline \multirow[t]{3}{*}{ Variables } & \multicolumn{6}{|c|}{ miR-1251-5p } \\
\hline & \multicolumn{3}{|c|}{ Premenopausal EC } & \multicolumn{3}{|c|}{ Postmenopausal EC } \\
\hline & Samples & Relative expression & $P$ & Samples & Relative expression & $P$ \\
\hline Yes & 2 & $\begin{array}{l}3.71 \times 10^{-5_{ \pm}} \\
1.41 \times 10^{-6}\end{array}$ & 0.312 & 3 & $\begin{array}{l}1.28 \times 10^{-} \\
2 \pm 5.18 \times 10^{-3}\end{array}$ & 0.734 \\
\hline No & 18 & $\begin{array}{l}1.11 \times 10^{-3} \pm \\
1.50 \times 10^{-3}\end{array}$ & & 37 & $\begin{array}{l}1.80 \times 10^{-} \\
2 \pm 2.24 \times 10^{-2}\end{array}$ & \\
\hline \multicolumn{7}{|c|}{ Hypertension } \\
\hline Yes & 3 & $\begin{array}{l}2.10 \times 10^{-2} \pm 2.10 \times 10^{-} \\
-\end{array}$ & \multirow[t]{2}{*}{0.093} & 18 & $\begin{array}{l}1.85 \times 10^{-} \\
2 \pm 2.47 \times 10^{-2}\end{array}$ & \multirow[t]{2}{*}{0.644} \\
\hline No & 17 & $\begin{array}{l}8.01 \times 10^{-3} \pm 1.31 \times 10^{-} \\
-\end{array}$ & & 22 & $\begin{array}{l}1.68 \times 10^{-} \\
2 \pm 1.92 \times 10^{-2}\end{array}$ & \\
\hline \multicolumn{7}{|l|}{ Diabetes } \\
\hline Yes & 2 & $\frac{1.10 \times 10^{-5} \pm 7.10 \times 10^{-}}{}$ & \multirow[t]{2}{*}{0.168} & 9 & $\begin{array}{l}3.41 \times 10^{-} \\
2 \pm 2.94 \times 10^{-2}\end{array}$ & \multirow[t]{2}{*}{0.031} \\
\hline No & 18 & $\frac{1.11 \times 10^{-2} \pm 1.50 \times 10^{-}}{}$ & & 31 & $\begin{array}{l}1.28 \times 10^{-} \\
2 \pm 1.64 \times 10^{-2}\end{array}$ & \\
\hline \multicolumn{7}{|c|}{ FIGO stage } \\
\hline I & 11 & $\begin{array}{l}8.22 \times 10^{-3} \pm 1.01 \times 10^{-} \\
2\end{array}$ & \multirow[t]{3}{*}{0.206} & 32 & $\begin{array}{l}1.82 \times 10^{-} \\
2 \pm 2.15 \times 10^{-2}\end{array}$ & \multirow[t]{3}{*}{0.501} \\
\hline II & 5 & $\frac{1.27 \times 10^{-2} \pm 1.87 \times 10^{-}}{}$ & & 4 & $\begin{array}{l}5.43 \times 10^{-} \\
{ }^{ \pm} \pm 3.00 \times 10^{-3}\end{array}$ & \\
\hline III & 4 & $\begin{array}{l}1.13 \times 10^{-2} \pm 2.26 \times 10^{-} \\
-\end{array}$ & & 4 & $\begin{array}{l}2.49 \times 10^{-} \\
2 \pm 3.15 \times 10^{-2}\end{array}$ & \\
\hline \multicolumn{7}{|l|}{ Grading } \\
\hline G1 & 9 & ]$_{3}^{1.10 \times 10^{-2} \pm 9.45 \times 10^{-}}$ & \multirow[t]{2}{*}{0.031} & 6 & $\begin{array}{l}1.11 \times 10^{-} \\
2 \pm 8.93 \times 10^{-3}\end{array}$ & \multirow[t]{2}{*}{0.638} \\
\hline G2 & 9 & $2^{1.10 \times 10^{-3} \pm 2.00 \times 10^{-}}$ & & 19 & $\begin{array}{l}2.25 \times 10^{-} \\
2 \pm 2.62 \times 10^{-2}\end{array}$ & \\
\hline
\end{tabular}




\begin{tabular}{|c|c|c|c|c|c|c|}
\hline \multirow[t]{3}{*}{ Variables } & \multicolumn{6}{|c|}{ miR-1251-5p } \\
\hline & \multicolumn{3}{|c|}{ Premenopausal EC } & \multicolumn{3}{|c|}{ Postmenopausal EC } \\
\hline & Samples & Relative expression & $\mathbf{P}$ & Samples & Relative expression & $\mathbf{P}$ \\
\hline G3 & 2 & $\begin{array}{l}2.90 \times 10^{-9} \pm 1.00 \times 10^{-} \\
10\end{array}$ & & 15 & $\begin{array}{l}1.40 \times 10^{-} \\
2 \pm 1.79 \times 10^{-2}\end{array}$ & \\
\hline \multicolumn{7}{|c|}{ Myometrial infiltration } \\
\hline$<1 / 2$ & 13 & ${ }_{2}^{1.12 \times 10^{-2} \pm 1.38 \times 10^{-}}$ & \multirow[t]{2}{*}{0.046} & 25 & $\begin{array}{l}1.99 \times 10^{-} \\
2 \pm 2.34 \times 10^{-2}\end{array}$ & \multirow[t]{2}{*}{0.476} \\
\hline$\geq 1 / 2$ & 7 & $\begin{array}{l}7.72 \times 10^{-3} \pm 1.68 \times 10^{-} \\
-\end{array}$ & & 15 & $\begin{array}{l}1.38 \times 10^{-} \\
2 \pm 1.82 \times 10^{-2}\end{array}$ & \\
\hline \multicolumn{7}{|c|}{ Lymph node metastasis } \\
\hline Yes & 2 & $\begin{array}{l}4.56 \times 10^{-2} \pm 7.07 \times 10^{-} \\
\end{array}$ & \multirow[t]{2}{*}{0.011} & 4 & $\begin{array}{l}2.49 \times 10^{-} \\
2 \pm 3.15 \times 10^{-2}\end{array}$ & \multirow[t]{2}{*}{0.983} \\
\hline No & 18 & $\begin{array}{l}6.00 \times 10^{-3} \pm 8.40 \times 10^{-} \\
4\end{array}$ & & 36 & $\begin{array}{l}1.68 \times 10^{-} \\
2 \pm 1.07 \times 10^{-2}\end{array}$ & \\
\hline \multicolumn{7}{|c|}{ Tumor metastasis(including cervix uteri, lymph node and other organs metastasis) } \\
\hline Yes & 9 & $2^{1.21 \times 10^{-2} \pm 1.91 \times 10^{-}}$ & \multirow[t]{2}{*}{0.824} & 9 & $\begin{array}{l}1.38 \times 10^{-} \\
2 \pm 2.21 \times 10^{-2}\end{array}$ & \multirow[t]{2}{*}{0.292} \\
\hline No & 11 & $\begin{array}{l}8.22 \times 10^{-3} \pm 1.01 \times 10^{-} \\
2\end{array}$ & & 31 & $\begin{array}{l}1.87 \times 10^{-} \\
2 \pm 2.16 \times 10^{-2}\end{array}$ & \\
\hline
\end{tabular}


Table 2

Relationship between miR-6892-5p expression and clinic pathological features of EC patients $(x \pm s)$

Variables

miR-6892-5p

Premenopausal EC

Samples Relative expression
Postmenopausal EC

P samples Relative expression P

Age at menarche (years)

\begin{tabular}{|c|c|c|c|c|c|}
\hline$\leq 12$ & 8 & $\begin{array}{l}2.51 \times 10^{-3} \pm \\
6.22 \times 10^{-3}\end{array}$ & 0.511 & 9 & $\begin{array}{l}1.39 \times 10^{-3} \pm \\
1.60 \times 10^{-3}\end{array}$ \\
\hline $\begin{array}{l}>12 \sim \leq \\
14\end{array}$ & 8 & $\begin{array}{l}6.64 \times 10^{-3} \pm \\
1.09 \times 10^{-2}\end{array}$ & & 9 & $\begin{array}{l}5.24 \times 10^{-3_{ \pm}} \\
8.57 \times 10^{-3}\end{array}$ \\
\hline$>14$ & 4 & $\begin{array}{l}5.59 \times 10^{-4_{ \pm}} \\
5.41 \times 10^{-4}\end{array}$ & & 22 & $\begin{array}{l}3.14 \times 10^{-3} \pm \\
4.16 \times 10^{-3}\end{array}$ \\
\hline
\end{tabular}

Menstrual cycle

$\begin{array}{lllll}\text { Regularity } 8 & 2.78 \times 10^{-3_{ \pm}} & 0.347 & 40 & *_{-} \\ & 6.13 \times 10^{-3} & & & \end{array}$

Irregularity 12

$4.44 \times 10^{-3} \pm 9.29 \times 10^{-}$

0

3

BMI

$<24$

3

$1.20 \times 10^{-3} \pm$

$0.004 \quad 10$

$1.37 \times 10^{-3} \pm$

0.638

$2.97 \times 10^{-4}$

$9.40 \times 10^{-4}$

$\geq 24 \sim<\quad 4$

28

4

$1.28 \times 10^{-2} \pm$

$1.32 \times 10^{-2}$

27

$3.97 \times 10^{-3} \pm$

$6.11 \times 10^{-3}$

$\geq 28$

$1.58 \times 10^{-3_{ \pm}}$

$4.90 \times 10^{-3}$

$\begin{array}{ll}3 & 2.56 \times 10^{-3} \pm \\ & 2.10 \times 10^{-3}\end{array}$

Pregnancies

\begin{tabular}{|c|c|c|c|c|c|}
\hline $0 \sim 1$ & 5 & $\begin{array}{l}3.26 \times 10^{-4} \pm 3.30 \times 10^{-} \\
4\end{array}$ & 0.092 & 12 & $\begin{array}{l}1.56 \times 10^{-3} \pm \\
1.93 \times 10^{-3}\end{array}$ \\
\hline$\geq 2$ & 15 & $\begin{array}{l}4.02 \times 10^{-3} \pm 9.04 \times 10^{-} \\
3\end{array}$ & & 28 & $\begin{array}{l}3.93 \times 10^{-3} \pm \\
5.93 \times 10^{-3}\end{array}$ \\
\hline
\end{tabular}

Family history of cancer

(Note: * The menstrual cycle of all the postmenopausal women is regular. Mann-Whitney U test) 


\begin{tabular}{|c|c|c|c|c|c|c|}
\hline \multirow[t]{3}{*}{ Variables } & \multicolumn{6}{|c|}{ miR-6892-5p } \\
\hline & \multicolumn{3}{|c|}{ Premenopausal EC } & \multicolumn{3}{|c|}{ Postmenopausal EC } \\
\hline & Samples & Relative expression & $\mathbf{P}$ & samples & Relative expression & $P$ \\
\hline Yes & 2 & $\begin{array}{l}8.97 \times 10^{-5_{ \pm}} \\
1.40 \times 10^{-9}\end{array}$ & 0.443 & 3 & $\begin{array}{l}1.84 \times 10^{-} \\
{ }^{ \pm} \pm 2.72 \times 10^{-3}\end{array}$ & 0.626 \\
\hline No & 18 & $\begin{array}{l}4.18 \times 10^{-3} \pm \\
8.38 \times 10^{-3}\end{array}$ & & 37 & $\begin{array}{l}3.33 \times 10^{-} \\
{ }^{ \pm} \pm 5.32 \times 10^{-3}\end{array}$ & \\
\hline \multicolumn{7}{|c|}{ Hypertension } \\
\hline Yes & 3 & $\frac{2.22 \times 10^{-2} \pm 3.70 \times 10^{-}}{3}$ & \multirow[t]{2}{*}{0.006} & 18 & $\begin{array}{l}5.19 \times 10^{-} \\
3_{ \pm} 7.17 \times 10^{-3}\end{array}$ & \multirow[t]{2}{*}{0.369} \\
\hline No & 17 & $\begin{array}{l}5.30 \times 10^{-4} \pm 5.40 \times 10^{-} \\
4\end{array}$ & & 22 & $\begin{array}{l}1.60 \times 10^{-} \\
{ }^{ \pm} \pm 1.30 \times 10^{-3}\end{array}$ & \\
\hline \multicolumn{7}{|l|}{ Diabetes } \\
\hline Yes & 2 & $\begin{array}{l}6.87 \times 10^{-4} \pm 1.50 \times 10^{-} \\
9\end{array}$ & \multirow[t]{2}{*}{0.798} & 9 & $\begin{array}{l}5.45 \times 10^{-} \\
{ }^{ \pm} \pm 5.81 \times 10^{-3}\end{array}$ & \multirow[t]{2}{*}{0.083} \\
\hline No & 18 & $\begin{array}{l}4.12 \times 10^{-3} \pm 8.41 \times 10^{-} \\
-\end{array}$ & & 31 & $\begin{array}{l}2.57 \times 10^{-} \\
{ }^{ \pm} \pm 4.87 \times 10^{-3}\end{array}$ & \\
\hline \multicolumn{7}{|c|}{ FIGO stage } \\
\hline I & 11 & $\begin{array}{l}5.14 \times 10^{-3} \pm 9.48 \times 10^{-} \\
3\end{array}$ & \multirow[t]{3}{*}{0.206} & 32 & $\begin{array}{l}2.30 \times 10^{-} \\
{ }^{ \pm} \pm 3.60 \times 10^{-3}\end{array}$ & \multirow[t]{3}{*}{0.040} \\
\hline II & 5 & $\begin{array}{l}1.72 \times 10^{-4} \pm 1.18 \times 10^{-} \\
4\end{array}$ & & 4 & $\begin{array}{l}1.15 \times 10^{-} \\
2 \pm 1.01 \times 10^{-2}\end{array}$ & \\
\hline III & 4 & $\begin{array}{l}4.54 \times 10^{-3} \pm 8.90 \times 10^{-} \\
3\end{array}$ & & 4 & $\begin{array}{l}2.25 \times 10^{-} \\
{ }^{ \pm} \pm 2.52 \times 10^{-3}\end{array}$ & \\
\hline \multicolumn{7}{|l|}{ Grading } \\
\hline G1 & 9 & $\begin{array}{l}6.17 \times 10^{-3} \pm 1.03 \times 10^{-} \\
2\end{array}$ & \multirow[t]{2}{*}{0.008} & 6 & $\begin{array}{l}6.21 \times 10^{-} \\
4_{ \pm 6.24 \times 10^{-4}}\end{array}$ & \multirow[t]{2}{*}{0.052} \\
\hline G2 & 9 & $2.20 \times 10^{-3} \pm 5.89 \times 10^{-}$ & & 19 & $\begin{array}{l}2.92 \times 10^{-} \\
{ }^{3} \pm 4.49 \times 10^{-3}\end{array}$ & \\
\hline
\end{tabular}




\begin{tabular}{|c|c|c|c|c|c|c|}
\hline \multirow[t]{3}{*}{ Variables } & \multicolumn{6}{|c|}{ miR-6892-5p } \\
\hline & \multicolumn{3}{|c|}{ Premenopausal EC } & \multicolumn{3}{|c|}{ Postmenopausal EC } \\
\hline & Samples & Relative expression & $\mathbf{P}$ & samples & Relative expression & $\mathbf{P}$ \\
\hline G3 & 2 & $\begin{array}{l}8.57 \times 10^{-5} \pm 1.00 \times 10^{-} \\
10\end{array}$ & & 15 & $\begin{array}{l}4.63 \times 10^{-} \\
{ }^{ \pm} \pm 6.55 \times 10^{-3}\end{array}$ & \\
\hline \multicolumn{7}{|c|}{ Myometrial infiltration } \\
\hline$<1 / 2$ & 13 & $\begin{array}{l}2.44 \times 10^{-3} \pm 6.59 \times 10^{-} \\
3\end{array}$ & \multirow[t]{2}{*}{0.841} & 25 & $\begin{array}{l}3.84 \times 10^{-} \\
{ }^{ \pm} \pm 6.34 \times 10^{-3}\end{array}$ & \multirow[t]{2}{*}{0.834} \\
\hline$\geq 1 / 2$ & 7 & $\begin{array}{l}6.26 \times 10^{-3} \pm 1.03 \times 10^{-} \\
2\end{array}$ & & 15 & $\begin{array}{l}2.17 \times 10^{-} \\
3_{ \pm} \pm 1.82 \times 10^{-3}\end{array}$ & \\
\hline \multicolumn{7}{|c|}{ Lymph node metastasis } \\
\hline Yes & 2 & $\begin{array}{l}8.99 \times 10^{-3} \pm 1.26 \times 10^{-} \\
2\end{array}$ & \multirow[t]{2}{*}{0.949} & 4 & $\begin{array}{l}2.25 \times 10^{-} \\
3_{ \pm} 2.52 \times 10^{-3}\end{array}$ & \multirow[t]{2}{*}{0.652} \\
\hline No & 18 & $\begin{array}{l}3.20 \times 10^{-3} \pm 7.69 \times 10^{-} \\
3\end{array}$ & & 36 & $\begin{array}{l}3.32 \times 10^{-} \\
{ }^{ \pm} \pm 5.39 \times 10^{-3}\end{array}$ & \\
\hline \multicolumn{7}{|c|}{ Tumor metastasis(including cervix uteri, lymph node and other organs metastasis) } \\
\hline Yes & 9 & $\begin{array}{l}2.11 \times 10^{-3} \pm 5.92 \times 10^{-} \\
3\end{array}$ & \multirow[t]{2}{*}{0.005} & 9 & $\begin{array}{l}6.27 \times 10^{-} \\
{ }^{ \pm} \pm 8.14 \times 10^{-3}\end{array}$ & \multirow[t]{2}{*}{0.178} \\
\hline No & 11 & $\begin{array}{l}5.14 \times 10^{-3} \pm 9.48 \times 10^{-} \\
3\end{array}$ & & 31 & $\begin{array}{l}2.33 \times 10^{-} \\
{ }^{3} \pm 3.66 \times 10^{-3}\end{array}$ & \\
\hline
\end{tabular}


Table 3

Clinical and pathological features between Premenopausal EC and Postmenopausal EC

\begin{tabular}{|c|c|c|c|c|}
\hline \multicolumn{2}{|l|}{ Variables } & $\begin{array}{l}\text { Premenopausal EC } \\
\text { (20 cases) }\end{array}$ & $\begin{array}{l}\text { Postmenopausal EC } \\
\text { (40 cases) }\end{array}$ & $\mathbf{P}$ \\
\hline \multicolumn{2}{|l|}{ Age at menarche ${ }^{1}$ (years) } & $13.10 \pm 1.62$ & $14.97 \pm 1.25$ & 0.000 \\
\hline \multirow[t]{2}{*}{ menstrual cycle ${ }^{2}$} & Regularity & $8(40 \%)$ & $40(100 \%)$ & \multirow[t]{2}{*}{0.000} \\
\hline & Irregularity & $12(60 \%)$ & $0(0 \%)$ & \\
\hline \multicolumn{2}{|l|}{ Pregnancies $^{1}$} & $2.35 \pm 1.31$ & $2.23 \pm 1.25$ & 0.062 \\
\hline \multirow[t]{3}{*}{$\mathrm{BMI}^{2}$} & $<24$ & $3(15 \%)$ & $10(25 \%)$ & \multirow[t]{3}{*}{0.000} \\
\hline & $\geq 24 \sim<28$ & $4(20 \%)$ & $27(67.5 \%)$ & \\
\hline & $\geq 28$ & $13(65 \%)$ & $3(7.5 \%)$ & \\
\hline \multirow[t]{2}{*}{ Family history of cancer ${ }^{2}$} & Yes & $2(10 \%)$ & $3(7.5 \%)$ & \multirow[t]{2}{*}{1.000} \\
\hline & No & $18(90 \%)$ & $37(92.5 \%)$ & \\
\hline \multirow[t]{2}{*}{ Hypertension ${ }^{2}$} & Yes & $3(15 \%)$ & $18(45 \%)$ & \multirow[t]{2}{*}{0.022} \\
\hline & No & $17(85 \%)$ & $22(55 \%)$ & \\
\hline \multirow[t]{2}{*}{ Diabetes $^{2}$} & Yes & $2(10 \%)$ & $9(22.5 \%)$ & \multirow[t]{2}{*}{0.409} \\
\hline & No & $18(90 \%)$ & $31(77.5 \%)$ & \\
\hline \multirow[t]{3}{*}{ FIGO Stage $^{1}$} & 口 & $11(55 \%)$ & $32(80 \%)$ & \multirow[t]{3}{*}{0.052} \\
\hline & 口 & $5(25 \%)$ & $4(10 \%)$ & \\
\hline & $\square$ & $4(20 \%)$ & $4(10 \%)$ & \\
\hline \multirow[t]{3}{*}{ Grading $^{1}$} & $\mathrm{G} 1$ & $9(45 \%)$ & $6(15 \%)$ & \multirow[t]{3}{*}{0.004} \\
\hline & $\mathrm{G} 2$ & $9(45 \%)$ & $19(47.5 \%)$ & \\
\hline & G3 & $2(10 \%)$ & $15(37.5 \%)$ & \\
\hline \multirow[t]{2}{*}{ Myometrial infiltration ${ }^{2}$} & $<1 / 2$ & $13(65 \%)$ & $25(62.5 \%)$ & \multirow[t]{2}{*}{0.850} \\
\hline & $\geq 1 / 2$ & $7(35 \%)$ & $15(37.5 \%)$ & \\
\hline \multirow[t]{2}{*}{ Lymph node metastasis ${ }^{2}$} & Yes & $2(10 \%)$ & $4(10 \%)$ & \multirow[t]{2}{*}{1.000} \\
\hline & No & $18(90 \%)$ & $36(90 \%)$ & \\
\hline
\end{tabular}

(Note :1.Mann-Whitney U test, 2.Pearson Chi-square test.) 


\begin{tabular}{|llll|}
\hline Variables & $\begin{array}{l}\text { Premenopausal EC } \\
(\mathbf{2 0} \text { cases })\end{array}$ & $\begin{array}{l}\text { Postmenopausal EC } \\
(\mathbf{4 0} \text { cases })\end{array}$ & P \\
\hline Recurrence $^{2}$ & $3(5 \%)$ & 0 & 0.492 \\
\hline (Note :1.Mann-Whitney U test, 2.Pearson Chi-square test.) & & \\
\hline
\end{tabular}

\section{Figures}

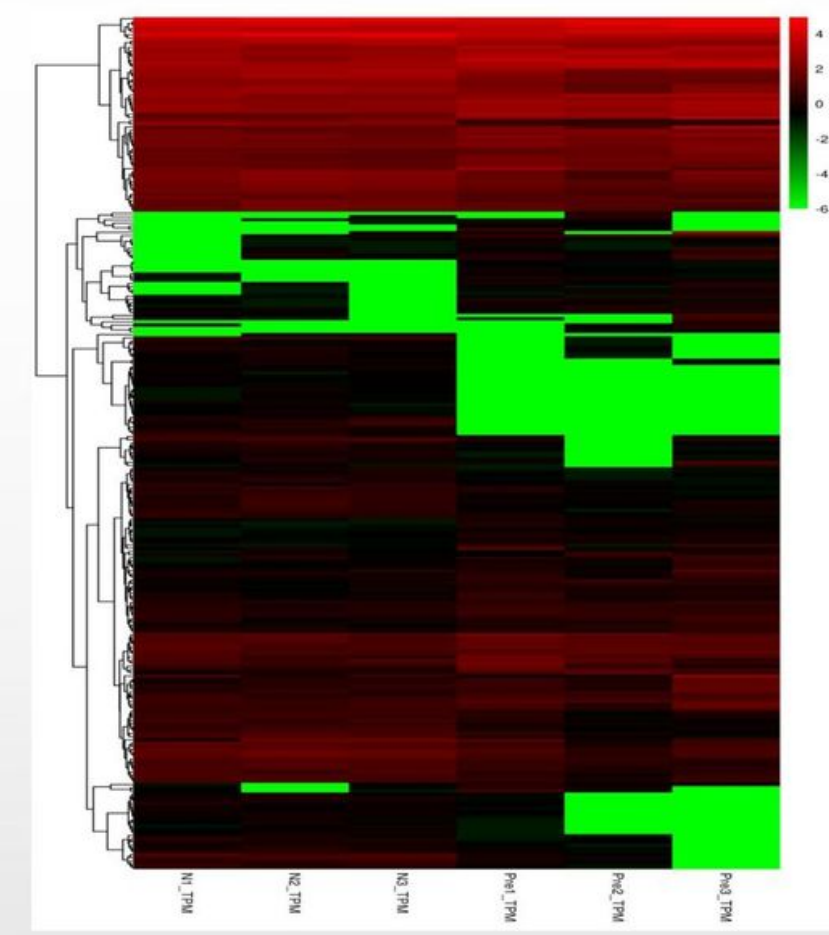

A

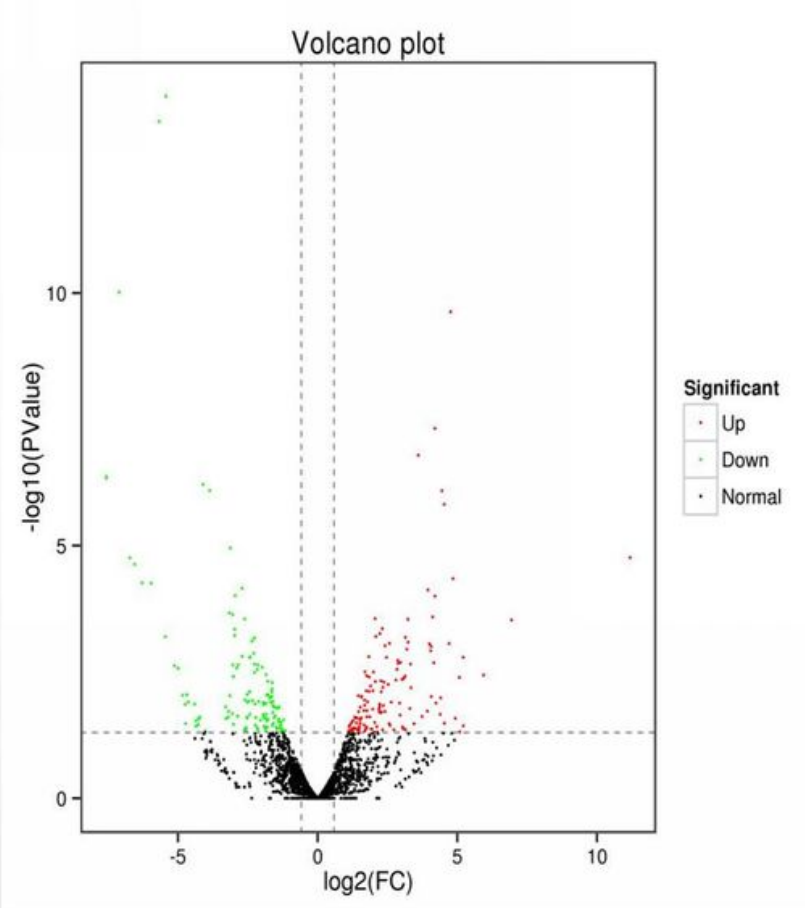

B

\section{Figure 1}

Heatmap $\triangle A$ ) and Volcano Plot(B) of differentially expressed miRNAs between 3 premenopausal EC tissue samples and 3 premenopausal normal endometrial tissue samples. Note:(A) Diflerent rows represent different miRNAs,color represents the level of expression of miRNAs in sample quantity log!0 (miRNA + 0.000001). (B) Each point in the figure represents a miRNA. The abscissa represents the logarithm of the differential multiple of the expression of a miRNA in two samples and the y-coordinate is the negative logarithm of pvalue. The green points in the figure represent down-regulated differentially expressed miRNAs, the red points represent up-regulated differentially expressed miRNAs, and the black points represent non-diflerentially expressed miRNAs. 
A

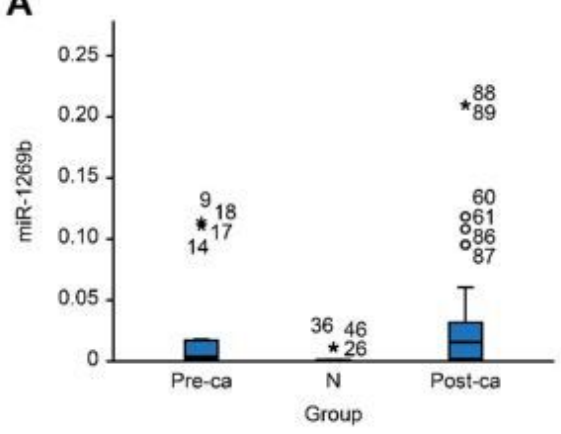

C

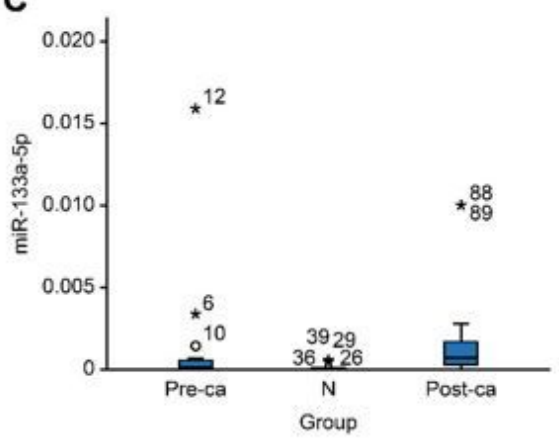

E

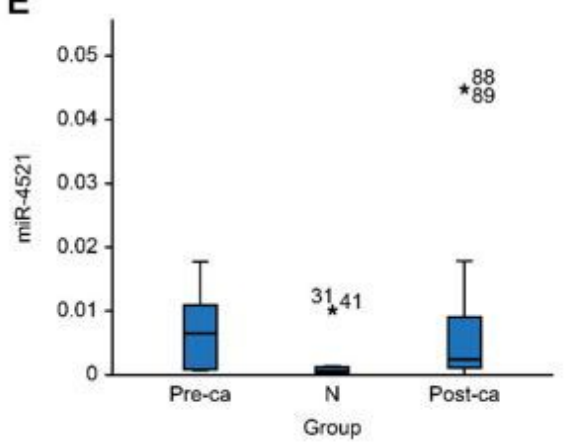

G

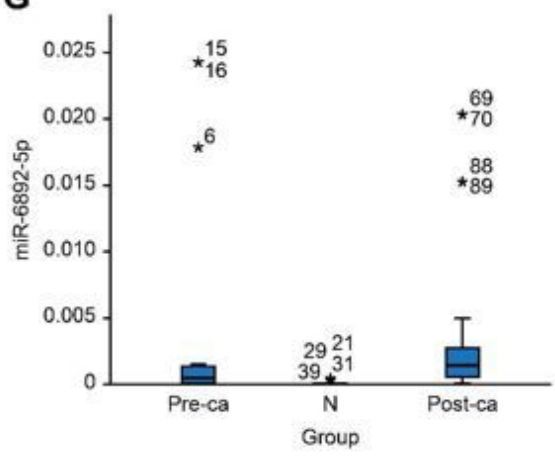

B

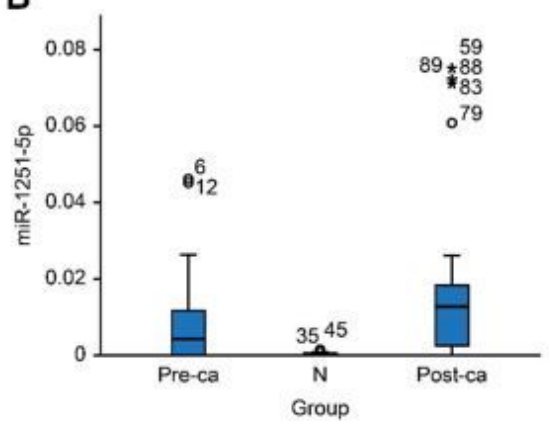

D

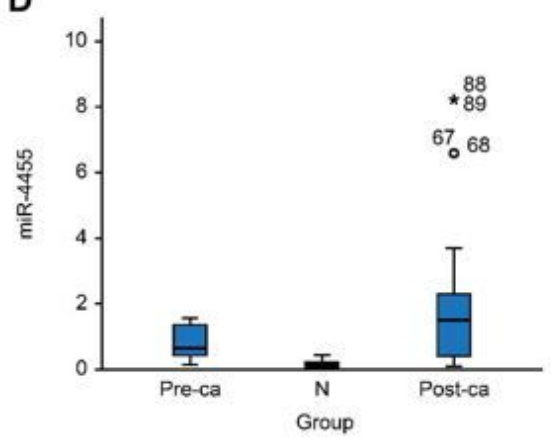

F

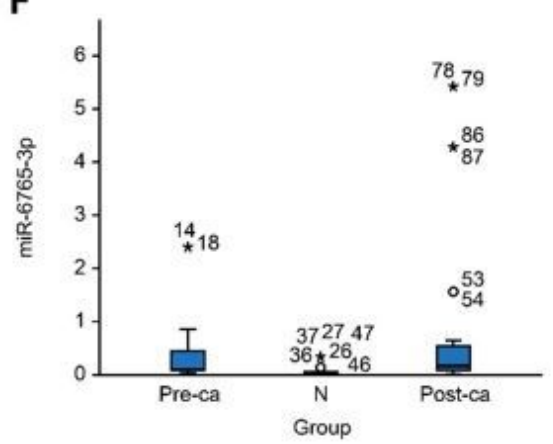

\section{Figure 2}

The screened miRNAs were relatively expressed in different groups of endometrial tissues. 


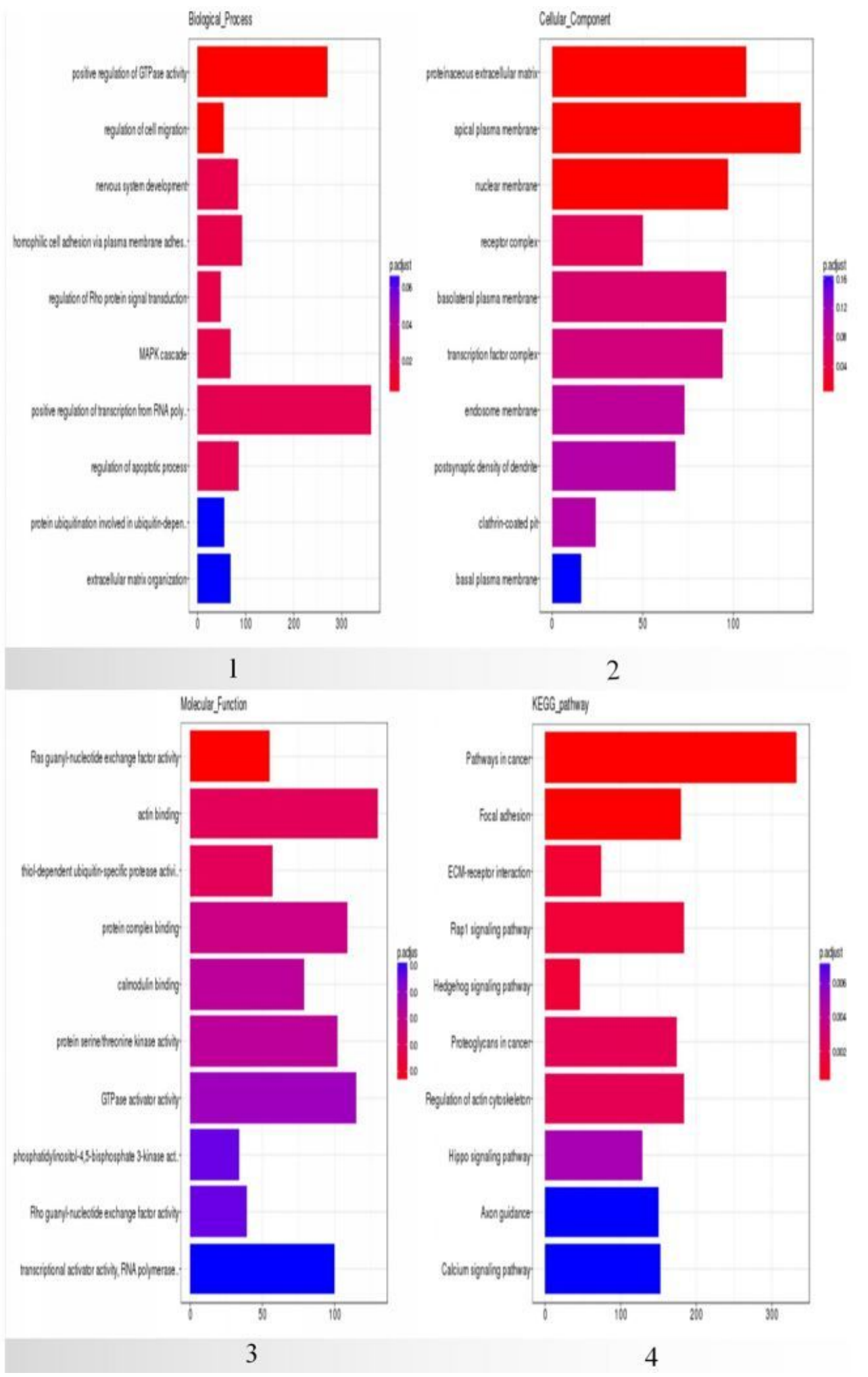

\section{Figure 3}

The top 10 of differentially expressed miRNA target gene GO enrichment bar graph. Note: The horizontal axis represents the number of genes in the pathway with differentially expressed miRNA target gene annotation, the vertical axis represents the pathway, and the color of the column represents the corrected $P$ value. 


\section{Supplementary Files}

This is a list of supplementary files associated with this preprint. Click to download.

- Fig.S1.jpg

- SupplementarytableSP.doc 